\title{
Growth mode evolution during homoepitaxy of GaAs (001)
}

\author{
M. D. Johnson, J. Sudijono, A. W. Hunt, and B. G. Orr \\ The Harrison M. Randall Laboratory, University of Michigan, Ann Arbor, Michigan 48109-1120
}

(Received 27 September 1993; accepted for publication 22 November 1993)

\begin{abstract}
Scanning tunneling microscopy studies have been performed on GaAs homoepitaxial films grown by molecular-beam epitaxy. After an initial transient regime, indicated by reflection high-energy electron diffraction oscillations, the system evolves to a dynamical steady state. This state is characterized by a constant step density and as such the growth mode can be termed generalized step flow.
\end{abstract}

Thin film deposition has become a critical technology for the advancement of modern electronics. A large number of artificial heterostructures have been produced by using various growth techniques. Molecular beam epitaxy (MBE) is particularly important because it affords monoatomic layer thickness control over films growing from the vapor phase at rclatively low temperatures under supersaturation conditions. ${ }^{1}$ With careful choice of the growth parameters to control the surface kinetics, one can create multilayered structures in which individual layers maintain their chemical integrity and form compositionally abrupt interfaces with one another.

Crucial to the perfection of the sample surface is the growth mode of the film. MBE growth is typically characterized by two types of two-dimensional modes: layer-bylayer and step flow. The controlling parameter which distinguishes these modes is the ratio of the typical separation between nucleating islands and the average terrace width. If the island separation is greater than the terrace width, the growth will be by step flow. Conversely, if the island separation is smaller than the terrace width, the growth will involve island nucleation and coalescence. The transition between these modes can be controlled by altering the growth rate, As/Ga flux ratio, substrate temperature, or the sample miscut.

In this letter we concentrate on conditions in which growth initiates in the layer-by-layer mode. One signature of this mode is the observation of reflection high-energy electron diffraction (RHEED) intensity oscillations. These oscillations indicate a temporal cycle of island formation and coalescence on the surface. ${ }^{2-4}$ Additionally, when beams scattered from adjacent layers are in phase, these intensity oscillations have been shown to be well correlated with the step density ${ }^{5}$ on the growing surface. ${ }^{6,7,12}$ This can be understood by noting that diffuse scattering occurs predominately at steps so that with the terrace interference removed by being at the Bragg diffraction condition, the RHEED specular intensity should vary inversely with the diffuse scattering or step density.

The goal of this letter is to understand the origin of the decay in the amplitude of RHEED intensity oscillations. In accord with the step density model, for the oscillations to die out the density of steps must evolve to a constant. What is the corresponding surface morphology for this steady state? It was suggested ${ }^{8}$ that the decay of the oscillations was due to an increase in surface width or number of incomplete lay- ers. By examining scanning tunneling microscopy (STM) images of the surface quenched at different points during growth, we will show a dynamical growth mode transition from layer to layer in the initial stages to a steady state characterized by a highly ramified geometry and a constant density of steps. The interface width remains relatively constant throughout this evolution.

The experiment consists of initiating growth from a recovered surface and then terminating deposition at a specific point during the growth process and transferring in situ into the STM. The quench procedure has been described previously. ${ }^{2,9}$ This process is then repeated for various termination points. Because the samples are removed from the STM for regrowth, no direct comparison can be made between any specific feature in the progression of the images. We have imaged the surface at multiple sites on multiple samples and the images shown are representative of the surface on a scale less than $0.5 \mu \mathrm{m}$.

Deposition was performed in a standard ultrahigh vacuum system, basc pressure $7 \times 10^{-11} \mathrm{~T}$. Effusion cells were used to produce both the $\mathrm{Ga}$ and $\mathrm{As}_{4}$ fluxes. Commercial $\mathrm{GaAs}(001)$ substrates were first chemically cleaned then loaded in the vacuum system where the oxide was removed at $580^{\circ} \mathrm{C}$ under an $\mathrm{As}_{4}$ flux. Prior to the experiments a 300 nm-thick buffer layer was grown. The substrate temperature during deposition was $555^{\circ} \mathrm{C}$. The As to $\mathrm{Ga}$ pressure ratio was 15 and the deposition rate was $0.18 \mu \mathrm{m} / \mathrm{h}$. The sample miscut as determined by STM was approximately $0.15^{\circ}$. The direction and magnitude of the local vicinality was found to vary appreciably.

We have previously published STM data contrasting the morphology of the film surface at it evolves from a RHEED maximum to a RHEED minimum. ${ }^{2}$ To summarize, the surface quenched at an intensity maximum shows a low density of monolayer holes and islands occurring in approximately equal numbers, while the surface quenched at a RHEED minimum shows many two-dimensional islands on the terraces and no holes. This is exactly the morphological progression expected for layer-by-layer growth.

As the deposition continues, the RHEED oscillations decay. Figure 1(a) shows the surface after 60 monolayers have been deposited and the RHEED oscillation amplitude has decayed to less than $5 \%$ of its original value. The surface displays a complicated geometry where the terrace edges have become so ramified that the vicinality of the sample is difficult to recognize. Figure 1 (b) shows the surface after 


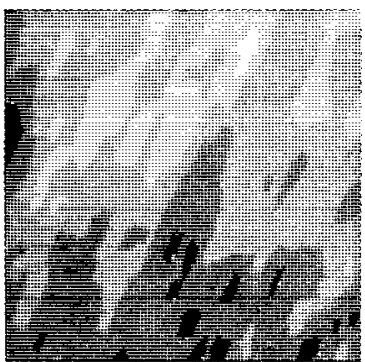

(a)

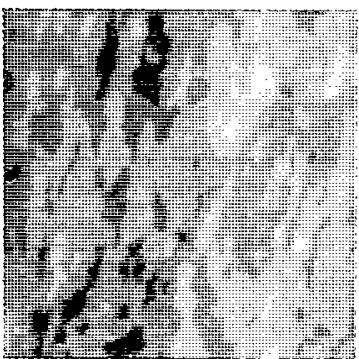

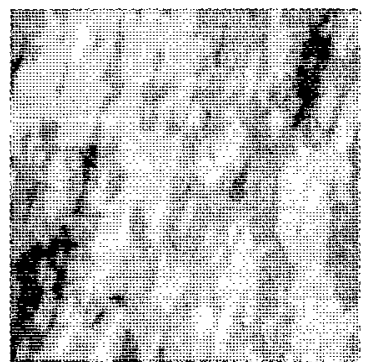

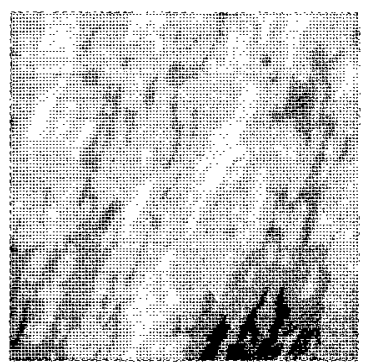

(d) (a)

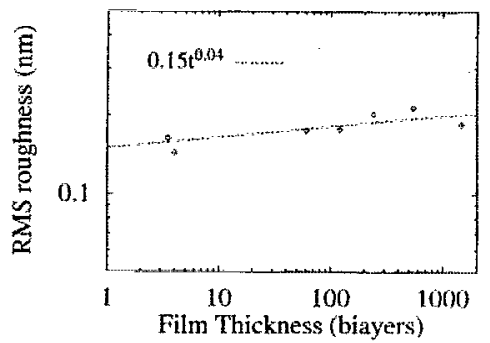

(b)

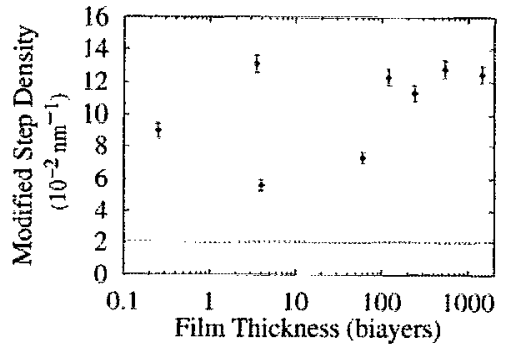

FIG. 1. STM images of GaAs deposited on a GaAs(001) buffer layer. The size of all images is $200 \mathrm{~nm} \times 200 \mathrm{~nm}$. The tunneling voltage $\left(V_{i}\right)$ was +2.8 $V_{3}$ applied to the sample, and the tumeling current $\left(I_{t}\right)$ was $80 \mathrm{pA}$. (a), (b), (c), and (d) were taken after deposition of $60,120,240$, and 1450 monolayers, respectivaly.

deposition of 120 monolayers. 'The surface in Fig. 1(a) has coarsened so much that it starts to nucleate two-dimensional istands on top of the terraces. Nevertheless, the overall morphology remains flat, with about four layers present. The STM images obtained after depositing 240 and 1450 monolayers [Figs. 1(c) and 1(d), respectively] show essentially identical topography to that of the 120 monolayer film. Surprisingly, further growth has not increased the rms surface roughness on the length scale of these images $(200 \mathrm{~nm})$. This is consistent with observations of large scale features in films grown by MBE. ${ }^{10}$ After the growth has reached 120 monolayers, the interface width of the surface increases extremely slowly, and the step density remains constant, see Fig. 2 . This points to a central feature of the data, that is the decay of the oscillation amplitude has occurred without an increase in the interface roughness. Further, the morphology has achieved a steady state configuration, through a balance of island nucleation and coalescence, and terrace step flow. This surface morphology evolves with a constant step density.

The growth of the film has changed from the initial layer-by-layer mode to something rather different. It is important to note that the film surface is still two dimensional and that the substrate temperature and sample miscut have remained constant throughout the deposition. In other words, the growth mode has been dynamically altered. This new mode is characterized by a balance of island coalescence, hole occlusion, and terrace propagation in just the proper combination so that the step density remains constant in time. We propose that this new mode be termed generalized step flow due to the constancy of the step density. This new mode should be contrasted with growth without the nucleation of islands, the traditional step flow mode, which is expected to have a much more uniform surface morphology.
FIG. 2. (a) rms roughness (in nm) of the surfaces as a function of deposition time (monolayer units). The rms roughness is defined as $\sqrt{1 / N \Sigma_{i}\left(h_{i}-h\right)^{2}}$, where $h_{i}$ is the height and the sum is over a $200 \mathrm{~nm} \times 200 \mathrm{~nm}$ area. (b) Plot of the surface step densities (in units of $10^{-2} \mathrm{~nm}-2$ ) as a function of thicknesses (in monolayers). The modified step density excludes the contribution from small holes less than $5 \mathrm{~nm}$ in diameter. The dashed line represents the step density of the starting surface.

The possibility exists however, that even traditional step flow growth is characterized by a more complicated dynamics than the uniform progression of a regular step train.

What is the physical mechanism responsible for the surface evolving to one with a constant step density? In this steady state all length scales up to a given size are represented. ${ }^{11}$ When an atom is deposited in a random position on the surface, the probability that it will combine with other adatoms to nucleate a new island is a function of the distance to the nearest step, either island or terrace. The closer the step, the less likely it will be for the adatom to nucleate a new island instead of being incorporated into the step. If the adatom nucleates an island then it creates step density where there was little before, and if it reaches a step edge then step density is either decreased or unchanged. Step density is decreased during hole occlusion and island coalescence and remains unchanged for propagation of terraces. By these competing processes the system has a self-regulating mechanism that keeps the step density constant on a large scale.

The imporlanl quantity to determine in order to study this mechanism is the distance an adatom must travel to reach the nearest step. We have examined the data in the following manner: for every point on the surface we find the distance to the nearest step, $d$, by examining outwardly in concentric circles. This is similar to the trajectory of a random walking adatom, which on average will fill a circular

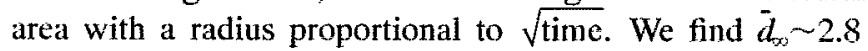
$\mathrm{nm}$ to be quite constant $(2.8,3.1,2.4$, and $2.7 \mathrm{~nm})$ from 


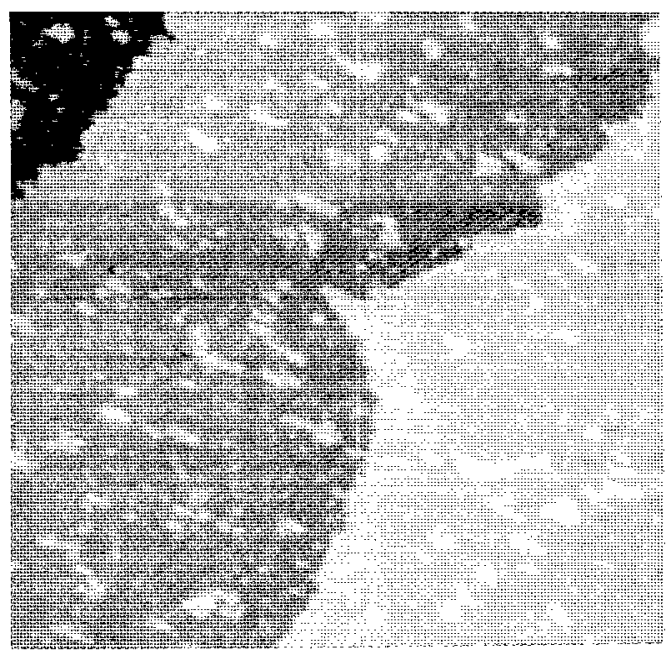

FIG. 3. STM image of 0.25 monolayers deposited on a GaAs(001) buffer layer. The sean range is $200 \mathrm{~nm} \times 200 \mathrm{~nm}$. The tunneling voltage $\left(V_{s}\right)$ was $+2.8 \mathrm{~V}$, applied to the sample, and the tunneling current $\left(I_{t}\right)$ was $80 \mathrm{pA}$. The average distance between nucleating islands, $\sigma$ is measured by counting the number, $n$ of islands in a given area, $L^{2}$, i.e., $\sigma=\sqrt{L^{2} / n}$.

images obtained from $120,240,540$, and 1450 layers, respectively. The specific step arrangements are different, however the same length scale is found for each surface.

We now seek to relate this distance $\bar{d}_{\infty}$ to a length scale set at the onset of deposition. One way to measure such a characteristic distance is to determine $\sigma$, the island spacing during the initial stages of growth on a singular substrate. ${ }^{.2}$ Figure 3 shows an STM image of 0.25 monolayer film deposited at $555^{\circ} \mathrm{C}$ on a buffer layer with a miscut of $0.15^{\circ}$. The small bright dots are islands all of which are larger than four $(2 \times 4)$ unit cells or $32 \mathrm{Ga}$ atoms. We measure $\sigma$ by counting the number of islands, $n$ in a given area $L^{2}$ so that $\sigma=\sqrt{I^{2} / n}$; we find $\sigma=16.5 \pm 2.0 \mathrm{~nm}$.

The growth mode transition follows from the relationship between $\sigma$ and $\bar{d}$. Initially $\bar{d}$ is very large, $\left(\vec{d}_{0}-25 \mathrm{~nm}\right)$ as the surface is nominally singular. As the growth proceeds by island formation $\vec{d}$ oscillates with large amplitude. However, additional step length is generated during growth and the amplitude decreases. When $\bar{l}$ becomes time invariant and smaller than $\sigma / 4,,^{13}$ the growth mode changes from island nucleation and coalescence to a generalized step flow. With further growth $\ddot{d}$ remains constant. From our data $\bar{d}_{x}=2.8$ $\mathrm{nm}$ and $\sigma / 4=4.1 \mathrm{~nm}$ which shows very good agreement with the prediction $\bar{d}_{\infty} \leqslant \sigma / 4$.

Although this growth mode does not allow one to predict the details of the complicated steady state surface morphology [Figs. 1(b)-1(d)], it does support a prediction about the asymptotic step density of a surface growing in this mode. A simple geometric model of a surface comprised of a rectangular array of terraces spaced so that the conditions of the generalized step flow are met will have a surface step density $\rho$, given by $\rho \simeq 2 / \sigma$. From our data, with $\sigma=16 \mathrm{~nm}$ the model would predict $\rho=0.125 \mathrm{ntm}^{-1}$. This can be compared with the measured value of asymptotic step density show in Fig. 2(b). The agreement is very good. Therefore, as a direct consequence of this growth model, if the early stage nucleation properties of a system are known, i.e., $\sigma$, then the late stage steady state step density is determined.

In summary, we have found a dynamical transition from island nucleation and coalescence to step flow growth and have measured a characteristic distance for the surface in the steady state which quantifies the tendency of the system to maintain a constant step density. However, this type of growth is not what is conventionally called step flow, i.e., a uniform progression of the steps across the surface. This classic step flow occurs when the substrate has a sutficient density of steps due to a large miscut, and the adatoms have a large enough mobility. The new feature presented here is that deposition has dynamically generated a morphology with sufficient step density to allow for the change from layer-by-layer growth to a generalized step flow growth mode. The model also provides a connection between the early stage nucleation properties and the asymptotic step density.

This work has been supported by Grant Nos. N0001489-J-1519 and NSF/DMR-8857828.

${ }^{1}$ B. A. Joyed, Contemp. Phys. 32, 21 (1991), and references thercin.

${ }^{2}$ J. Sudijono, M. D. Johnson, C. W. Snyder, M. B. Elowitz, and B. G, Orr, Phys. Rev. Lett. 69, 2811 (1992).

3 J. A. Strocio, D. T. Pleree, and R. A, Dragoset, Phys, Rev. Lett. 70, 3615 (1993).

${ }^{4}$ A. V. Latyshev, A. L. Aseev, A. B. Krasilnikov, and S. I. Stenin, Phys. Status Solidi A 113, 421 (1989).

step density is defined as the step length of adatoms, holes, islands, and terraces per unit area.

${ }^{5}$ S. Clarke and D. D. Vvedensky, Phys. Rev. Lett. 58, 2235 (1987).

${ }^{7}$ T. Shitara, D. D. Vvedensky, M. R. Wilby, J. Zhang, J. H. Neave, and B. A. Joyce, Appl. Ploys, 1.ett. 60, 1504 (1992).

${ }^{8}$ H. C. Kang and J. W. Evans, Suri. Sci. 271, 321 (1992).

${ }^{9}$ B. G. Orr, C. W. Snyder, and M. D. Johnson, Rev. Sci. Instrum. 6, 1400 (1991).

${ }^{10}$ M. D. Johnson, C. Orme, A. W. Hunt, D. Graff, I. Sudijono, L. M. Sander, and B. G. Orr, Phys, Rev, Lett. (to be published); C. Orme, M. D. Johnson, J. Sudijono, K. 'I. Leung, B. G. Orr, Appl. Phys. Lett. (to be published); G. W. Sinith, A. J. Pidduck, C. R. Whitehouse, J. L. Glasper, A. M. Keir, and C. Pickering, ibid. 59, 3282 (1991); T. Tiedje (private communication).

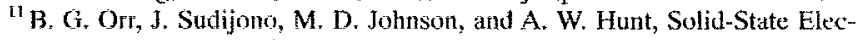
tron. (to be published).

12 J. Villain, A. Pimpinelli, L. Tang, and D. Wolf, J. Phys. I 2, 2107 (1992).

13 The number 4 in the expression is a geometrical factor, 